\title{
Construction of admissible linear orders for pairs of intervals
}

\author{
L. De Miguel ${ }^{1}$ H.Bustince ${ }^{1}$ E. Induráin ${ }^{1}$ A. Kolesárová ${ }^{2}$ R. Mesiar $^{2,3}$ B. De Baets ${ }^{4}$ \\ ${ }^{1}$ Universidad Publica de Navarra, Campus Arrosadía s/n 31006 Spain \\ 2 Slovak University of Technology in Bratislava, Radlinskeho 9-11, Bratislava, Slovakia \\ 3 University of Ostrava, Dvořákova 7, 70103 Ostrava 1, Czech Republic \\ ${ }^{4}$ Ghent University, Coupure links 653, B-9000 Gent, Belgium
}

\begin{abstract}
In this work we construct linear orders between pairs of intervals by using aggregation functions. We apply these orders in a decision-making problem where the experts provide their opinions by means of interval-valued fuzzy sets.
\end{abstract}

Keywords: Interval-valued fuzzy sets, decision making, linear order.

\section{Introduction}

The membership degree of each element of a fuzzy set is a value in the interval $[0,1]$ (linear ordered set). Then, given $x, y \in[0,1]$ such that $x \neq y$, $x<y$ or $y<x$. This property, inherited of real numbers, could be a crucial fact in many applications where a ranking between alternatives or membership degrees must be calculated. Moreover, some families of aggregation functions, such as OWA operators and Choquet integrals, require a linear order in their definitions.

In some applications, such as decision making, it has been proven that the systematic use of extensions of fuzzy sets is a useful tool [1]. This is the case, for instance, of interval-valued fuzzy sets $[2,3]$ or, equivalently, Atanassov intuitionistic fuzzy sets [4]. However, in all these extensions, the membership degrees are evaluated in a poset (partially order set) and the problem of incomparability between alternatives may arise.

To avoid this incomparability problem, in this paper we propose a method to generate linear orders between pairs of intervals and we apply it in a decision making problem. In this way, we select as the best option the alternative which is associated to the largest pair of intervals, with respect to the considered linear order.

As in decision making problems we must also aggregate the information furnished by the experts by means of aggregation functions $[5,6,7]$, we have decided to use these functions to generate the orders.

All these considerations have led us to aim at the following objectives:

(1) To use aggregation functions for building linear orders for pairs of intervals whose endpoints belong to the unit interval;

(2) To apply it in a decision making problem.

The structure of the paper is the following. In Section 2 we introduce the notation and recall some well-known notions. In Section 3 we construct linear orders between pairs of intervals through aggregation functions. Section 4 contains an application of the theoretical results in a multi-expert decision making problem. Some concluding remarks and future research lines close the paper.

\section{Previous concepts and results}

We start by recalling some well-known concepts that will be useful for subsequent developments throughout the paper.

\subsection{On orders and partially ordered sets}

Definition 2.1 A partial order $\preceq$ over $P$ is a binary relation which is reflexive, antisymmetric and transitive. If $\preceq$ is a partial order, the pair $(P, \preceq)$ is called a partially ordered set (poset).

Given a poset $(P, \preceq)$, and $x, y \in P$ we say that $x$ and $y$ are comparable if $x \preceq y$ or $y \preceq x$.

Besides, we call

a) $1_{P}$, the top of the poset, if for all $x \in P$ it holds $x \preceq 1_{P}$;

b) $0_{P}$, the bottom of the poset, if for all $x \in P$ it holds $0_{P} \preceq x$.

Notice that, in case they exist, $1_{P}$ and $0_{P}$ are unique.

Let $K([0,1]) \subset \mathbb{R}^{2}$ be given by

$$
K([0,1])=\{(\underline{x}, \bar{x}) \in[0,1] \times[0,1] \mid \underline{x} \leq \bar{x}\},
$$

and let $L([0,1])$ be the set of all closed subintervals of the unit interval, that is

$L([0,1])=\{\mathbf{x} \mid \mathbf{x}=[\underline{x}, \bar{x}]$ such that $0 \leq \underline{x} \leq \bar{x} \leq 1\}$.

There is a straightforward bijection

$i: K([0,1]) \longrightarrow L([0,1])$ given by

$i((\underline{x}, \bar{x}))=[\underline{x}, \bar{x}]=\mathbf{x}$.

Through this bijection, the partial order on $\mathbb{R}^{2}$, $(a, b) \preceq_{2}(c, d)$ if and only if $a \leq c$ and $b \leq d$ induces an equivalent partial order on $L([0,1])$, namely, 
$\mathbf{x} \preceq_{2} \mathbf{y}$ if and only if $\underline{x} \leq \underline{y}$ and $\bar{x} \leq \bar{y}$.

In this way, $\left(L([0,1]), \preceq_{2}\right)$ is a poset whose bottom and top are, respectively, $\mathbf{0}=[0,0]$ and $\mathbf{1}=$ $[1,1]$. In fact, the bijection $i$ is a lattice isomorphism $^{1}$.

We refer as $(L([0,1]))^{2}$ to the set of pairs of closed subintervals of the unit interval; that is,

$(L([0,1]))^{2}=\{([\underline{x}, \bar{x}],[\underline{y}, \bar{y}])$ with $\underline{x}, \bar{x}, \underline{y}, \bar{y} \in[0,1]\}$.

Similarly to the case of $\mathbb{R}^{2}$ and $L([0,1])$, the partial order on $\mathbb{R}^{4}$, namely,

$\left(a_{1}, b_{1}, c_{1}, d_{1}\right) \preceq_{4}\left(a_{2}, b_{2}, c_{2}, d_{2}\right)$ if and only if

$a_{1} \leq a_{2}$ and $b_{1} \leq b_{2}$ and $c_{1} \leq c_{2}$ and $d_{1} \leq d_{2}$,

also induces an equivalent partial order $\preceq_{4}$ on $(L([0,1]))^{2}$, given by

$$
\begin{gathered}
\left(\mathbf{x}_{\mathbf{1}}, \mathbf{y}_{\mathbf{1}}\right) \preceq_{4}\left(\mathbf{x}_{\mathbf{2}}, \mathbf{y}_{\mathbf{2}}\right) \text { if and only if } \underline{x}_{1} \leq \underline{x}_{2} \text { and } \\
\bar{x}_{1} \leq \bar{x}_{2} \text { and } \underline{y}_{1} \leq \underline{y}_{2} \text { and } \bar{y}_{1} \leq \bar{y}_{2}
\end{gathered}
$$

In this way, $\left((L([0,1]))^{2}, \preceq_{4}\right)$ becomes a poset whose bottom and top are, respectively,

$$
(\mathbf{0}, \mathbf{0})=([0,0],[0,0]) \text { and }(\mathbf{1}, \mathbf{1})=([1,1],[1,1]) .
$$

Example 2.1 Let the pairs of intervals be

- $z_{1}=([0.05,0.2],[0.1,0.4])$,

- $z_{2}=([0.3,0.7],[0.4,0.6])$,

- $z_{3}=([0.1,0.6],[0.5,0.95])$, and

- $z_{4}=([0.5,0.9],[0.2,0.7])$.

The elements $z_{i}$ can be represented in the unit square $[0,1]^{2}$ as in Fig. 1. In this Figure each pair of intervals is drawn as a rectangle for which the first interval lies in the horizontal axis and the second interval lies in the vertical one. The advantage of this representation is that some visual interpretations can be drawn. For example, we have that the intervals of $z_{3}$ and $z_{4}$ are wider than those of $z_{1}$ and $z_{2}$, since their areas are significantly greater. Besides, $z_{i} \preceq_{4} z_{j}$ if and only if each corner of the rectangle $z_{i}$ is located below and on the left side of its corresponding corner in the rectangle $z_{j}$. For example, in Fig 1 we know that $z_{1} \preceq_{4} z_{i}$ for $i \in\{2,3,4\}$. Similarly, we can deduct that $z_{2}, z_{3}$ and $z_{4}$ are incomparable in terms of $\preceq_{4}$.

Definition 2.2 A linear order $\leq$ over $P$ is a binary relation which is transitive, antisymmetric and total. Equivalently, a linear order is a partial order under which every pair of elements is comparable.

\footnotetext{
${ }^{1}$ The sets $K([0,1])$ and $L([0,1])$ have already been used, suitably equipped with some order and latticial structure $[8,9]$, to construct some universal codomain where it was possible to represent different kinds of orderings as, e.g., total preorders, interval-orders and semiorders, by means of a single function that preserves the ordinal structure. The bijection $i: K([0,1]) \longrightarrow L([0,1])$ has also been considered in those approaches, and some other similar bijections and/or latticial isomorphims as well as order isotonies have also been introduced accordingly. Other possible universal codomain to represent different kinds of orderings, which is essentially equivalent to $K([0,1])$ consists of triangular and symmetric fuzzy numbers. For further information see $[10,11,12,13]$.
}

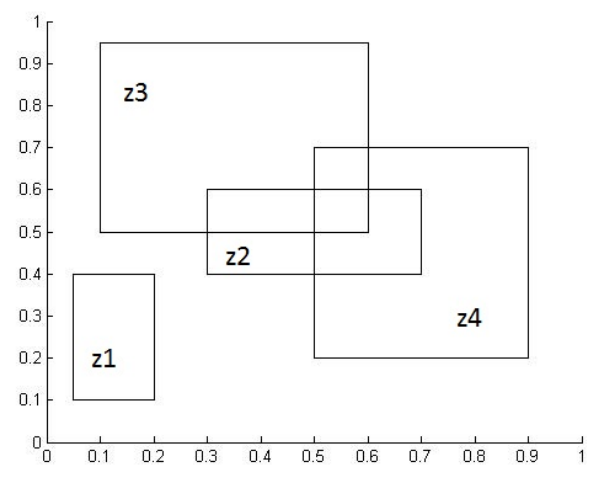

Figure 1: Pairs of intervals

Example 2.2 Some examples of linear orders on $L([0,1])$ are the lexicographic orders, given by:

- (lexicographic-1 order) $\boldsymbol{x} \leq_{\text {lex1 }} \boldsymbol{y}$ if and only if

$-(\underline{x}<\underline{y}) ;$ or

$-(\underline{x}=\underline{y}$ and $\bar{x} \leq \bar{y})$

- (lexicographic-2 order) $\boldsymbol{x} \leq_{\text {lex2 }} \boldsymbol{y}$ if and only if

$-(\bar{x}<\bar{y}) ;$ or

$-(\bar{x}=\bar{y}$ and $\underline{x} \leq \underline{y})$

In [14], a special class of linear orders is defined.

Definition 2.3 [14] An order $\leq$ on $L([0,1])$ is said to be admissible if it is linear and refines the partial order $\preceq_{2}$, i.e., it is a linear order satisfying that for all $\boldsymbol{x}, \boldsymbol{y} \in L([0,1])$ such that $(\underline{x}, \bar{x}) \preceq_{2}(\underline{y}, \bar{y})$, it holds $\boldsymbol{x} \leq \boldsymbol{y}$.

These admissible orders were introduced to deal with interval-valued fuzzy sets in applications (see [15]), since for those sets membership degrees are given in terms of intervals. Notice that lexicographic orders are particular instances of admissible orders.

\subsection{Fuzzy sets and extensions of fuzzy sets}

Since Zadeh introduced [16] the concept of a fuzzy set many extensions of the latter have been defined in order to deal with imprecision in different settings and problems. We recall here the definition of fuzzy sets and some of the most commonly used extensions.

Definition 2.4 [16] A fuzzy set $A$ on a universe $U$ is a mapping $A: U \longrightarrow[0,1]$, where $A(u)$ denotes the membership degree of the element $u$ to the fuzzy set $A$.

Definition 2.5 [17] An interval-valued fuzzy set $A$ on a universe $U$ is a mapping $A: U \longrightarrow L([0,1])$, where $A(u)$ denotes the membership degree of the element $u$ to the interval-valued fuzzy set $A$. 
Definition 2.6 [18] A set-valued fuzzy set (SVFS) $A$ on a universe $U$ is a mapping $A: U \longrightarrow$ $\mathcal{P}([0,1]) \backslash \emptyset$, where $\mathcal{P}([0,1])$ is the power set and $A(u)$ denotes the membership degree of the element $u$ to the SVFS A.

\subsection{Aggregation functions}

In many problems, it is necessary to fuse or aggregate inputs or data. A common tool to do so is provided by aggregation functions.

Definition 2.7 An aggregation function $M$ is a mapping $M:[0,1]^{n} \rightarrow[0,1]$ for some $n \geq 2$, that satisfies:

- $M(0, \ldots, 0)=0, \quad M(1, \ldots, 1)=1$, and

- For any pair $\left(x_{1}, \ldots, x_{n}\right)$ and $\left(y_{1}, \ldots, y_{n}\right)$ of $n$-tuples such that

$x_{i}, y_{i} \in[0,1]$ with $x_{i} \leq y_{i}$ for all $i \in\{1, \ldots, n\}$, then $M\left(x_{1}, \ldots, x_{n}\right) \leq M\left(y_{1}, \ldots, y_{n}\right)$;

that is, $M$ is monotonically increasing in each one of its arguments.

For further information and some generalizations of these functions see [19].

A relevant example of aggregation function is provided by triangular norms $[5,7,20,21]$.

Definition 2.8 A function $T:[0,1]^{2} \rightarrow[0,1]$ is called a triangular norm (t-norm for short) if it is symmetric, associative, increasing with respect to the order $\leq$ and $T(x, 1)=x$ for all $x \in[0,1]$.

Finally, aggregation functions have been used to generate admissible orders.

Proposition 2.1 [14] Let $B_{1}, B_{2}:[0,1]^{2} \rightarrow[0,1]$ be two continuous aggregation functions, such that for all $\left(p_{1}, p_{2}\right),\left(q_{1}, q_{2}\right) \in K([0,1])$, the equalities $B_{1}\left(p_{1}, p_{2}\right)=B_{1}\left(q_{1}, q_{2}\right)$ and $B_{2}\left(p_{1}, p_{2}\right)=B_{2}\left(q_{1}, q_{2}\right)$ only hold provided that $\left(p_{1}, p_{2}\right)=\left(q_{1}, q_{2}\right)$.

The order $\leq_{B_{1}, B_{2}}$ on $L([0,1])$, given by

$$
\begin{gathered}
\boldsymbol{x} \leq_{B_{1}, B_{2}} \boldsymbol{y} \text { if and only if } B_{1}(\underline{x}, \bar{x})<\left(B_{1}(\underline{y}, \bar{y})\right) \text { or } \\
\left(B_{1}(\underline{x}, \bar{x})=B_{1}(\underline{y}, \bar{y}) \text { and } B_{2}(\underline{x}, \bar{x}) \leq B_{2}(\underline{y}, \bar{y})\right),
\end{gathered}
$$

is an admissible order on $L([0,1])$.

\section{Admissible orders on $(L([0,1]))^{2}$}

By means of the partial order $\preceq_{4}$ the problem of incomparability between two elements on $(L([0,1]))^{2}$ can arise. Besides, some special classes of aggregations, such as Choquet and Sugeno integrals actually require a linear order. In this section, we construct linear orders on $(L([0,1]))^{2}$ generalizing the concept of admissible orders on $L([0,1])$ given in Def. 2.3.
Definition 3.1 An order $\leq$ on $(L([0,1]))^{2}$ is an admissible order if it is linear order on $(L([0,1]))^{2}$ and refining the order $\preceq_{4}$ in Eq. (1). Namely, it is a linear order satisfying that for all $\left(\boldsymbol{x}_{1}, \boldsymbol{y}_{1}\right),\left(\boldsymbol{x}_{2}, \boldsymbol{y}_{2}\right) \in$ $(L([0,1]))^{2},\left(\boldsymbol{x}_{\boldsymbol{1}}, \boldsymbol{y}_{\mathbf{1}}\right) \preceq_{4}\left(\boldsymbol{x}_{2}, \boldsymbol{y}_{2}\right)$ implies $\left(\boldsymbol{x}_{1}, \boldsymbol{y}_{1}\right) \leq$ $\left(\boldsymbol{x}_{2}, \boldsymbol{y}_{2}\right)$.

The method introduced by Bustince et al. in [14] to build admissible order via aggregation functions can be generalized to handle elements in $(L([0,1]))^{2}$.

Proposition 3.1 Let $M=\left(M_{1}, M_{2}, M_{3}, M_{4}\right)$ be an ordered 4-tuple of aggregation functions $M_{i}:[0,1]^{4} \rightarrow[0,1]$ such that for all $(\boldsymbol{p}, \boldsymbol{q}),(\boldsymbol{r}, \boldsymbol{s}) \in(L([0,1]))^{2}$ the equalities $M_{i}(\underline{p}, \bar{p}, q, \bar{q})=M_{i}(\underline{r}, \bar{r}, \underline{s}, \bar{s})$ for all $i \in\{1,2,3,4\}$ only hold if $(\boldsymbol{p}, \boldsymbol{q})=(\boldsymbol{r}, \boldsymbol{s})$.

Then, an admissible order can be defined as follows:

$\left(\boldsymbol{x}_{1}, \boldsymbol{y}_{1}\right) \leq_{M}\left(\boldsymbol{x}_{2}, \boldsymbol{y}_{2}\right)$ if and only if one of the (mutually exclusive) following conditions is satisfied

i) $M_{1}\left(\underline{x}_{1}, \bar{x}_{1}, \underline{y}_{1}, \bar{y}_{1}\right)<M_{1}\left(\underline{x}_{2}, \bar{x}_{2}, \underline{y}_{2}, \bar{y}_{2}\right)$;

ii) $M_{1}\left(\underline{x}_{1}, \bar{x}_{1}, \underline{y}_{1}, \bar{y}_{1}\right)=M_{1}\left(\underline{x}_{2}, \bar{x}_{2}, \underline{y}_{2}, \bar{y}_{2}\right)$ and $M_{2}\left(\underline{x}_{1}, \bar{x}_{1}, \underline{y}_{1}, \bar{y}_{1}\right)<M_{2}\left(\underline{x}_{2}, \bar{x}_{2}, \underline{y}_{2}, \bar{y}_{2}\right)$;

iii) $M_{1}\left(\underline{x}_{1}, \bar{x}_{1}, \underline{y}_{1}, \bar{y}_{1}\right)=M_{1}\left(\underline{x}_{2}, \bar{x}_{2}, \underline{y}_{2}, \bar{y}_{2}\right)$ and $M_{2}\left(\underline{x}_{1}, \bar{x}_{1}, \underline{y}_{1}, \bar{y}_{1}\right)=M_{2}\left(\underline{x}_{2}, \bar{x}_{2}, \underline{y}_{2}, \bar{y}_{2}\right)$ and $M_{3}\left(\underline{x}_{1}, \bar{x}_{1}, \underline{y}_{1}, \bar{y}_{1}\right)<M_{3}\left(\underline{x}_{2}, \bar{x}_{2}, \underline{y}_{2}, \bar{y}_{2}\right) ;$

iv) $M_{1}\left(\underline{x}_{1}, \bar{x}_{1}, \underline{y}_{1}, \bar{y}_{1}\right)=M_{1}\left(\underline{x}_{2}, \bar{x}_{2}, \underline{y}_{2}, \bar{y}_{2}\right)$ and $M_{2}\left(\underline{x}_{1}, \bar{x}_{1}, \underline{y}_{1}, \bar{y}_{1}\right)=M_{2}\left(\underline{x}_{2}, \bar{x}_{2}, \underline{y}_{2}, \bar{y}_{2}\right)$ and $M_{3}\left(\underline{x}_{1}, \bar{x}_{1}, \underline{y}_{1}, \bar{y}_{1}\right)=M_{3}\left(\underline{x}_{2}, \bar{x}_{2}, \underline{y}_{2}, \bar{y}_{2}\right)$ and $M_{4}\left(\underline{x}_{1}, \bar{x}_{1}, \underline{y}_{1}, \bar{y}_{1}\right) \leq M_{4}\left(\underline{x}_{2}, \bar{x}_{2}, \underline{y}_{2}, \bar{y}_{2}\right)$.

Proof. The order $\leq_{M}$ refines $\preceq_{4}$ since every $M_{i}$ is an aggregation function. Besides, the linearity is assured since the four conditions only meet if $\left(\mathbf{x}_{1}, \mathbf{y}_{1}\right)=\left(\mathbf{x}_{2}, \mathbf{y}_{\mathbf{2}}\right)$. The transitivity follows from the transitivity of the standard order on $[0,1]$.

Remark 3.1 If we permute the aggregation functions $M_{i}$, the new tuple also satisfies the condition of Prop. 3.1. Then, another admissible order is constructed.

Henceforward, we only consider the order generated by four aggregation functions (in Prop 3.1), that we call 4-admissible order. Thus, all the ideas to be introduced till the end of the section refer to such family of admissible orders.

Example 3.1 The four projections could be used to construct the lexicographic orders.

1. The standard lexicographic order: let $M_{i}$ be the aggregation function that maps to the $i$-th component (i.e. the $i$-th projection). In that case, $\left(\boldsymbol{x}_{1}, \boldsymbol{y}_{1}\right) \leq_{M}\left(\boldsymbol{x}_{2}, \boldsymbol{y}_{2}\right)$ if and only if

- $\left(\underline{x}_{1}<\underline{x}_{2}\right)$, or

- $\left(\underline{x}_{1}=\underline{x}_{2}\right.$ and $\left.\bar{x}_{1}<\bar{x}_{2}\right)$, or

- $\left(\underline{x}_{1}=\underline{x}_{2}, \bar{x}_{1}=\bar{x}_{2}\right.$ and $\left.\underline{y}_{1}<\bar{y}_{2}\right)$, or

- $\left(\underline{x}_{1}=\underline{x}_{2}, \bar{x}_{1}=\bar{x}_{2}, \underline{y}_{1}=\underline{y}_{2}\right.$ and $\left.\bar{y}_{1} \leq \bar{y}_{2}\right)$. 
2. The reversed lexicographic order: let $M_{i}$ be the aggregation function that maps to the $(5-i)$-th component (i.e. the $(5-i)$-th projection). In that case, $\left(\boldsymbol{x}_{1}, \boldsymbol{y}_{1}\right) \leq_{A}\left(\boldsymbol{x}_{2}, \boldsymbol{y}_{2}\right)$ if and only if

- $\left(\bar{y}_{1}<\bar{y}_{2}\right)$, or

- $\left(\bar{y}_{1}=\bar{y}_{2}\right.$ and $\left.\underline{y}_{1}<\underline{y}_{2}\right)$, or

- $\left(\bar{y}_{1}=\bar{y}_{2}, \underline{y}_{1}=\underline{y}_{2}\right.$ and $\left.\bar{x}_{1}<\bar{x}_{2}\right)$, or

- $\left(\bar{y}_{1}=\bar{y}_{2}, \underline{y}_{1}=\underline{y}_{2}, \bar{x}_{1}=\bar{x}_{2}\right.$ and $\left.\underline{x}_{1} \leq \underline{x}_{2}\right)$.

3. Any other permutation of the projections gives rise to an admissible order where we compare the components in a predetermined order.

Proposition 3.2 Let $M=\left(M_{1}, M_{2}, M_{3}, M_{4}\right)$ be four aggregation functions given by

$$
M_{i}\left(\underline{x}_{1}, \bar{x}_{1}, \underline{y}_{1}, \bar{y}_{1}\right)=\alpha_{i} \underline{x}_{1}+\beta_{i} \bar{x}_{1}+\gamma_{i} \underline{y}_{1}+\delta_{i} \bar{y}_{1},
$$

with $\alpha_{i}, \beta_{i}, \gamma_{i}, \delta_{i} \in[0,1], \alpha_{i}+\beta_{i}+\gamma_{i}+\delta_{i}=1$ and

$$
|D|=\left|\begin{array}{llll}
\alpha_{1} & \beta_{1} & \gamma_{1} & \delta_{1} \\
\alpha_{2} & \beta_{2} & \gamma_{2} & \delta_{2} \\
\alpha_{3} & \beta_{3} & \gamma_{3} & \delta_{3} \\
\alpha_{4} & \beta_{4} & \gamma_{4} & \delta_{4}
\end{array}\right| \neq 0
$$

Then, the order generated by the aggregation functions $A_{i}$ is a 4-admissible order.

Proof. The functions $M_{i}$ are weighted arithmetic means. Let $\left(\underline{x}_{1}, \bar{x}_{1}, \underline{y}_{1}, \bar{y}_{1}\right),\left(\underline{x}_{2}, \bar{x}_{2}, \underline{y}_{2}, \bar{y}_{2}\right) \in$ $(L([0,1]))^{2}$, such that

$\alpha_{i} \underline{x}_{1}+\beta_{i} \bar{x}_{1}+\gamma_{i} \underline{y}_{1}+\delta_{i} \bar{y}_{1}=\alpha_{i} \underline{x}_{2}+\beta_{i} \bar{x}_{2}+\gamma_{i} \underline{y}_{2}+\delta_{i} \bar{y}_{2}$

for $i \in\{1, \ldots, 4\}$. Since $|D| \neq 0$, both linear systems have a unique and common solution, i.e., $\left(\underline{x}_{1}, \bar{x}_{1}, \underline{y}_{1}, \bar{y}_{1}\right)=\left(\underline{x}_{2}, \bar{x}_{2}, \underline{y}_{2}, \bar{y}_{2}\right)$. The result follows now from Prop. 3.1.

Example 3.2 Let $M$ contain the following aggregation functions:

- $M_{1}\left(\underline{x}_{1}, \bar{x}_{1}, \underline{y}_{1}, \bar{y}_{1}\right)=\frac{1}{5} \underline{x}_{1}+\frac{1}{5} \bar{x}_{1}+\frac{1}{5} \underline{y}_{1}+\frac{2}{5} \bar{y}_{1}$;

- $M_{2}\left(\underline{x}_{1}, \bar{x}_{1}, \underline{y}_{1}, \bar{y}_{1}\right)=\frac{3}{8} \underline{x}_{1}+\frac{3}{8} \bar{x}_{1}+\frac{1}{8} \underline{y}_{1}+\frac{1}{8} \bar{y}_{1}$;

- $M_{3}\left(\underline{x}_{1}, \bar{x}_{1}, \underline{y}_{1}, \bar{y}_{1}\right)=\frac{1}{4} \underline{x}_{1}+\frac{1}{4} \bar{x}_{1}+\frac{1}{4} \underline{y}_{1}+\frac{1}{4} \bar{y}_{1}$;

- $M_{4}\left(\underline{x}_{1}, \bar{x}_{1}, \underline{y}_{1}, \bar{y}_{1}\right)=0 \underline{x}_{1}+\frac{1}{2} \bar{x}_{1}+\frac{1}{2} \underline{y}_{1}+0 \bar{y}_{1}$.

Since $|D|=-0.0063$, the order generated by $M$, as in Prop. 3.1, is a 4-admissible order.

Remark 3.2 Notice that due to the fact that all the elements of the matrix are smaller than 1 the value of the determinant is close to 0.

If we consider $M$ as the combination of four vectors

$$
\begin{array}{r}
R=\left\{<\alpha_{1}, \beta_{1}, \gamma_{1}, \delta_{1}>,<\alpha_{2}, \beta_{2}, \gamma_{2}, \delta_{2}>\right. \\
\left.<\alpha_{3}, \beta_{3}, \gamma_{3}, \delta_{3}>,<\alpha_{4}, \beta_{4}, \gamma_{4}, \delta_{4}>\right\}
\end{array}
$$

the construction of admissible orders as in Prop. 3.2 has an interesting geometrical interpretation. First, the condition of Prop. 3.2 means that the vectors of $R$ must constitute a basis.

In this way, because of the restrictions in the selection of the weights of the means, given four directions on $\mathbb{R}^{4}$ that form a basis, up to a permutation of the directions there is a unique admissible order $\leq_{M}$ constructed through the four directions.

Finally, after changing the basis, the coordinates of the pairs of intervals in the new basis, (which are now in $\left.[0,1]^{4}\right)$, are ordered through the standard lexicographic order.

Proposition 3.3 Let $B_{i}:[0,1]^{2} \longrightarrow[0,1], i \in$ $\{1, \ldots, 4\}$ be four aggregation functions. If

- $M_{i}(\underline{x}, \bar{x}, \underline{y}, \bar{y})=B_{i}(\underline{x}, \bar{x})$ for $i \in\{1,2\}$, and

- $M_{j}(\underline{x}, \bar{x}, \underline{y}, \bar{y})=B_{j}(\underline{y}, \bar{y})$ for $j \in\{3,4\}$,

generate a 4-admissible order then the order that generates is $\left(\boldsymbol{x}_{1}, \boldsymbol{y}_{1}\right) \leq_{M}\left(\boldsymbol{x}_{2}, \boldsymbol{y}_{2}\right)$ if and only if

i) $\left(\boldsymbol{x}_{\boldsymbol{1}}<_{B_{1}, B_{2}} \boldsymbol{x}_{\boldsymbol{1}}\right)$, or

ii) $\left(\boldsymbol{x}_{\boldsymbol{1}}=\boldsymbol{x}_{2}\right.$ and $\left.\boldsymbol{y}_{1} \leq_{B_{3}, B_{4}} \boldsymbol{y}_{2}\right)$,

where $\leq_{B_{i}, B_{j}}$ is the order on $L([0,1])$ generated in Prop. 2.1.

Proof. It is straightforward.

Notice that, if we use the same order in both intervals, namely, $B_{1}=B_{3}$ and $B_{2}=B_{4}$, the result is a 4 -admissible order where we combine the lexicographic 1 order with the interval order $\leq_{B_{1}, B_{2}}$. For instance, the standard lexicographic order can be seen as the composition of the lexicographic-1 order between intervals combined with itself.

Alternatively, notice that, if

- $M_{i}(\underline{x}, \bar{x}, y, \bar{y})=B_{i}(y, \bar{y})$ for $i \in\{1,2\}$, and

- $M_{j}(\underline{x}, \bar{x}, \underline{y}, \bar{y})=B_{j}(\underline{x}, \bar{x})$ for $j \in\{3,4\}$,

then the resulting order is also a 4-admissible order.

Atanassov's operators $\mathbb{K}_{\alpha}$ are binary aggregation functions given by $\mathbb{K}_{\alpha}(a, b)=a+\alpha(b-a)$ with $a, b, \alpha \in[0,1]$.

In our particular case, since the inputs are intervals, an Atanassov's operator acting on the endpoints of the intervals yields a point inside the corresponding interval.

Example 3.3 Let $\alpha_{1}, \alpha_{2}, \alpha_{3}, \alpha_{4} \in[0,1]$, with $\alpha_{1} \neq$ $\alpha_{2}$ and $\alpha_{3} \neq \alpha_{4}$. Let $M=\left(M_{1}, M_{2}, M_{3}, M_{4}\right)$ be four aggregation functions given by

- $M_{i}\left(\underline{x}_{1}, \bar{x}_{1}, \underline{y}_{1}, \bar{y}_{1}\right)=\mathbb{K}_{\alpha_{i}}\left(\underline{x}_{1}, \bar{x}_{1}\right)$, for $i \in\{1,2\}$, and

- $M_{j}\left(\underline{x}_{1}, \bar{x}_{1}, \underline{y}_{1}, \bar{y}_{1}\right)=\mathbb{K}_{\alpha_{j}}\left(\underline{y}_{1}, \bar{y}_{1}\right)$, for $j \in\{3,4\}$.

The tuple $M$ generates the 4-admissible order:

$\left(\boldsymbol{x}_{1}, \boldsymbol{y}_{1}\right) \leq_{M}\left(\boldsymbol{x}_{2}, \boldsymbol{y}_{2}\right)$ if and only if

- $\left(\boldsymbol{x}_{1}<_{\mathbb{K}_{\alpha_{1}}, \mathbb{K}_{\alpha_{2}}} \boldsymbol{x}_{2}\right)$, or

- $\left(\boldsymbol{x}_{\boldsymbol{1}}=\mathbb{K}_{\alpha_{1}}, \mathbb{K}_{\alpha_{2}} \boldsymbol{x}_{2}\right.$ and $\left.\boldsymbol{y}_{1} \leq_{\mathbb{K}_{\alpha_{3}}, \mathbb{K}_{\alpha_{4}}} \boldsymbol{y}_{2}\right)$. 
From the construction in Example 3.3, we can retrieve some well-known orders. For example, if $\left\{\alpha_{1}, \alpha_{2}\right\}=\{0,1\}$ and $\left\{\alpha_{3}, \alpha_{4}\right\}=\{0,1\}$, we obtain lexicographic orders. Besides, all these 4-admissible orders are particular examples of the construction in Prop. 3.2, with $c=d=0$ for $M_{1}, M_{2}$ and $a=b=0$ for $M_{3}$ and $M_{4}$.

\section{A decision making problem}

Decision making problems may be summarized as follows. We have a set of $p$ alternatives:

$$
Z=\left\{z_{1}, \cdots, z_{p}\right\}
$$

and a set of $n>2$ experts:

$$
E=\left\{e_{1}, \cdots, e_{n}\right\}
$$

Each of the latter provides her/his preferences on the former set of alternatives by means of a preference relation in the following way:

$$
r_{e l}=\left(\begin{array}{cccc}
- & r_{(e l) 12} & \cdots & r_{(e l) 1 p} \\
r_{(e l) 21} & - & \cdots & r_{(e l) 2 p} \\
\cdots & \cdots & - & \cdots \\
r_{(e l) p 1} & \cdots & \cdots & -
\end{array}\right)
$$

Here $r_{(e l) i j}$, with $i \neq j$, expresses how much expert $l$ (with $l \in\{1, \cdots, n\}$ ) prefers the alternative $z_{i}$ over the alternative $z_{j}$.

The problem is to select an alternative which is optimal as regards the experts assessments.

In [20], it is stated that the resolution of a group decision making problem consists of two steps:

(1) Uniform representation of information. In this phase, the heterogeneous information for the problem (the information can be represented by means of preference orderings or utility functions or fuzzy preference relations) is translated into homogeneous information by means of different transformation functions (see [22]).

(2) Application of a selection procedure. This procedure consists of two phases:

(2.1) Aggregation phase. A collective preference structure is built from the set of individual homogeneous preference structures.

(2.2) Exploitation phase. A given method is applied to the collective preference structure to obtain a selection of alternatives.

In this case, we propose a decision making problem where a car has to be chosen between the four possible alternatives:
(1) $z_{1} \longrightarrow$ Ford,
(2) $z_{2} \longrightarrow$ Hyundai,
(3) $z_{3} \longrightarrow$ Renault,
(4) $z_{4} \longrightarrow$ Volvo.

A group of $n$ experts has been asked for providing their assessments by means of preference relations as in Eq. (2), where each element is an interval. Besides, they are asked to look at two different characteristics: price and quality. Then, each element of the matrices is composed of two intervals (the first interval expresses their preferences about price and the second about quality). In the aggregation phase, we have built the collective matrix preference aggregating the $n$ preference relations provided by the experts differentiating the two characteristics.

$$
\begin{gathered}
\text { Column } 1 \\
- \\
R_{c}=\left(\begin{array}{cc}
\text { Column } 2 \\
{[0,0.6],[0.3,0.6]} & {[0.3,0.3],[0.5,1]} \\
{[0.3,0.7],[0.2,0.8]} & - \\
{[0.5,0.8],[0.6,0.6]} & {[0,0.9],[0.2,0.4]} \\
\text { Column 3 } & \text { Column } 4 \\
{[0.5,0.6],[0.4,0.6]} & {[0.1,0.8],[0.1,0.3]} \\
{[0.2,0.6],[0.6,0.9]} & {[0.4,0.4],[0.8,1]} \\
- & {[0.1,0.6],[0.2,0.3]} \\
{[0.3,0.4],[0.8,1]} & -
\end{array}\right)
\end{gathered}
$$

We are going to use the voting method in the exploitation phase. This method consists in aggregating the values in each row of the collective matrix $R_{c}$ in such a way that, at the end, we have as many values (pairs of intervals) as rows. We will select the alternative associated to the largest pair, according to the considered linear order.

To aggregate we are going to use the geometric mean $G(\mathbf{x}, \mathbf{y}, \mathbf{z})=[\sqrt[3]{\underline{x} \underline{y} \underline{z}}, \sqrt[3]{\overline{x y z}}$.

Applying $G$ to each row of $R_{c}$ we get a new matrix, say $R g$ given by:

$$
R g=\left(\begin{array}{c}
{[0.2466,0.5241],[0.2714,0.5646]} \\
{[0,0.5241],[0.5241,0.8143]} \\
{[0.2289,0.7230],[0.2714,0.6214]} \\
{[0,0.6604],[0.4579,0.6214]}
\end{array}\right)
$$

In this setting, the elements are not comparable through the partial order. For this reason we are going to consider the admissible order $\leq_{A}$ defined through the following aggregation functions.

$$
\begin{aligned}
& -M_{1}\left(\underline{x}_{1}, \bar{x}_{1}, \underline{y}_{1}, \bar{y}_{1}\right)=\frac{8}{20} \underline{x}_{1}+\frac{8}{20} \bar{x}_{1}+\frac{2}{20} \underline{y}_{1}+\frac{2}{20} \bar{y}_{1} \\
& \text { - } M_{2}\left(\underline{x}_{1}, \bar{x}_{1}, \underline{y}_{1}, \bar{y}_{1}\right)=\frac{1}{20} \underline{x}_{1}+\frac{5}{20} \bar{x}_{1}+\frac{3}{20} \underline{y}_{1}+\frac{2}{20} \bar{y}_{1} \\
& \text { - } M_{3}\left(\underline{x}_{1}, \bar{x}_{1}, \underline{y}_{1}, \bar{y}_{1}\right)=\frac{1}{20} \underline{x}_{1}+\frac{10}{20} \bar{x}_{1}+\frac{8}{20} \underline{y}_{1}+\frac{1}{20} \bar{y}_{1} \\
& \text { - } M_{4}\left(\underline{x}_{1}, \bar{x}_{1}, \underline{y}_{1}, \bar{y}_{1}\right)=\frac{1}{4} \underline{x}_{1}+\frac{1}{4} \bar{x}_{1}+\frac{1}{4} \underline{y}_{1}+\frac{1}{4} \bar{y}_{1} .
\end{aligned}
$$

With this order, we have the following ranking

$$
z_{2} \leq_{M} z_{4} \leq_{M} z_{1} \leq_{M} z_{3}
$$

i.e.,

Hyundai $\leq_{M}$ Volvo $\leq_{M}$ Ford $\leq_{M}$ Renault 
and the selected car is Renault.

In some cases, the resulting order would be directly induced by the context of the problem. Otherwise, it may happen that for different admissible orders, we obtain different selections associated to the same decision making problem. For instance, in the particular example of cars if the composed lexicographic 1 order is used the solution would be Ford instead of Renault.

To cope with this situation we propose the following algorithm.

(1) To construct several linear orders built with the methods developed in the previous sections.

(2) For each order, to apply in the exploitation phase the voting method with the same aggregation. For instance, in out example we have used the geometric mean.

(3) To select the alternative which appears as the best placed in the majority of all the soobtained rankings.

In our considered problem, we choose as third order the one generated as Prop. 3.3 using the Xu $\mathrm{y}$ Yager interval order [23]. This order can be rewritten as an admissible order constructed through

- $B_{1}(u, v)=B_{3}(u, v)=\frac{u+v}{2}$

- $B_{2}(u, v)=B_{4}(u, v)=v$.

Then the best alternative is the third one, that is, we must choose Renault.

Clearly, the nature of the problem will impose the number of linear orders to be considered and/or the conditions that will force us to use alternative methods.

\section{Conclusions}

In this paper we have constructed linear orders between pairs of intervals in $(L([0,1]))^{2}$. Besides, we have applied these linear orders in a decision making problem. Anyway, they could be used in any application or theoretical development such as of aggregation functions where a ranking between pairs of intervals should be calculated.

\section{Acknowledgment}

The work has been supported by projects TIN201340765-P and MTM2012-37894-C02-02 of the Spanish Ministry of Science and the Research Services of the Universidad Publica de Navarra. The last two authors acknowledge the support of the grants VEGA 1/0419/13 and VEGA 1/0420/15.

\section{References}

[1] H. Bustince, E. Barrenechea, M. Pagola, J. Fernandez, Z.S. Xu, B. Bedregal, J. Montero, H. Hagras, F. Herrera, and B. De Baets. A historical account of types of fuzzy sets and their relationships. IEEE Trans. on Fuzzy Systems, 2015. In press.

[2] H. Bustince, E. Barrenechea, and M. Pagola. Generation of interval-valued fuzzy and Atanassov intuitionistic fuzzy connectives from fuzzy connectives and from $K_{\alpha}$ operators: laws for conjunctions and disjunctions, amplitude. International Journal of Intelligent Systems, 23(6):680-714, 2008.

[3] G. Deschrijver, C. Cornelis, and E.E. Kerre. On the representation of intuitionistic fuzzy tnorms and t-conorms. IEEE Trans. on Fuzzy Systems, 12(1):45-61, 2004.

[4] K. T. Atanassov. Intuitionistic fuzzy sets. Fuzzy Sets and Systems, 20(1):87-96, 1986.

[5] T. Calvo, A. Kolesárová, M. Komorníková, and R. Mesiar. Aggregation operators: properties, classes and construction methods. In T. Calvo, G. Mayor, and R. Mesiar, editors, Aggregation Operators, volume 97 of Studies in Fuzziness and Soft Computing, pages 3-104. PhysicaVerlag HD, 2002.

[6] G. Beliakov, H. Bustince, D.P. Goswami, U.K. Mukherjee, and N.R. Pal. On averaging operators for Atanassov's intuitionistic fuzzy sets. Information Sciences, 181(6):1116-1124, 2011.

[7] G. Beliakov, A. Pradera, and T. Calvo. Aggregation Functions: A Guide for Practitioners. Studies In Fuzziness and Soft Computing, Springer, 2007.

[8] G. Birkhoff. Lattice Theory. American Mathematical Society, 1967.

[9] H.A. Davey, B.A. Priestley. Introduction to Lattices and Order. Cambridge University Press, 2002.

[10] M. J. Campión, J. C. Candeal, and E. Induráin. Representability of binary relations through fuzzy numbers. Fuzzy Sets and Systems, 157(1):1-19, 2006.

[11] J.C. Candeal, J. Gutiérrez García, and E. Induráin. Universal codomains to represent interval orders. International Journal of Uncertainty, Fuzziness and Knowledge-Based Systems, 17(2):197-219, 2009.

[12] G. Bosi, J. Gutiérrez García, and E. Induráin. Unified representability of total preorders and interval orders through a single function: The lattice approach. Order, 26(3):255-275, 2009.

[13] G. Bosi. A numerical representation of semiorders on a countable set. Rivista di matematica per le scienze economiche e sociali, 16(2):15-19, 1993.

[14] H. Bustince, J. Fernandez, A. Kolesárová, and R. Mesiar. Generation of linear orders for intervals by means of aggregation functions. Fuzzy Sets and Systems, 220:69-77, 2013.

[15] H. Bustince, M. Galar, B. Bedregal, A. Kolesárová, and R. Mesiar. A new approach to interval-valued Choquet integrals and the problem of ordering in interval-valued 
fuzzy set applications. IEEE Trans. on Fuzzy Systems, 21(6):1150-1162, 2013.

[16] L. A. Zadeh. Fuzzy sets. Information and control, 8(3):338-353, 1965.

[17] R. Sambuc. Fonctions and floues: application a l'aide au diagnostic en pathologie thyroidienne. Faculté de Médecine de Marseille, 1975.

[18] I. Grattan-Guinness. Fuzzy membership mapped onto intervals and many-valued quantities. Mathematical Logic Quarterly, 22(1):149-160, 1976.

[19] M. Komorníková and R. Mesiar. Aggregation functions on bounded partially ordered sets and their classification. Fuzzy Sets and Systems, 175(1):48-56, 2011.

[20] J. Fodor and M. Roubens. Fuzzy Preference Modelling and Multicriteria Decision Support. In Theory and Decision Library, 1994.

[21] M. Grabisch, J.L. Marichal, R. Mesiar, and E. Pap. Aggregation Functions. Cambridge University Press, 2009.

[22] F. Chiclana, F. Herrera, and E. HerreraViedma. Integrating three representation models in fuzzy multipurpose decision making based on fuzzy preference relations. Fuzzy Sets and Systems, 97(1):33-48, 1998.

[23] Z. Xu and R.R. Yager. Some geometric aggregation operators based on intuitionistic fuzzy sets. International Journal of General Systems, 35(4):417-433, 2006. 